\title{
Study on the Phase Separation Process of Poly(methyl methacrylate)/ Poly(styrene-co-acrylonitrile) Blend by SR-SAXS
}

\author{
Kiyoshi Ikawa, ${ }^{\dagger}$ Akio Uemura, Satoru Hosoda, Toshiro Kojima, ${ }^{*}$ \\ Hideo NomUra, ${ }^{*}$ Kazuhiro ChIKaISHI, ${ }^{*}+\dagger$ and Yoshiyuki AmemiYa ${ }^{* *}$ \\ Sumitomo Chemical Co., Ltd., Chiba Research Laboratory, \\ 5-1 Anesakikaigan, Ichihara, Chiba 299-01, Japan \\ * Sumitomo Chemical Co., Ltd., Tsukuba Research Laboratory, \\ 6 Kitahara, Tsukuba, Ibaraki 300-32, Japan \\ ** National Laboratory for High Energy Physics, \\ 1-1 Oho, Tsukuba, Ibaraki 305, Japan
}

(Received February, 14, 1991)

\begin{abstract}
The early stage of spinodal decomposition of partially miscible poly(methyl methacrylate)/poly(styrene-co-acrylonitrile) blend was studied by time-resolved synchrotron radiation small-angle X-ray scattering (SR-SAXS). Intensity change of a scattering peak corresponding to the concentration fluctuation was traced after rapid heating to the two-phase region from the miscible state. At the initial stage, the dominant phase size was kept constant on the order of tens of nanometers, and then, grew to the order of sub-microns during decomposition. The apparent diffusion constant was determined according to the Cahn-Hilliard theory.

KEY WORDS Spinodal Decomposition / Polymer Blend / Poly(methyl methacrylate) / Poly(styrene-co-acrylonitrile) / Synchrotron RadiationSAXS /
\end{abstract}

Some investigations ${ }^{1-11}$ have been made on spinodal decompositon (SD) of partially miscible polymer blend systems out of interest in both basic research and industrial applications. Light scattering, electron microscopy etc. have played important roles in these investigations. Studies on the early stage of SD succeeded in verifying the application of the Cahn's theory ${ }^{12}$ to polymer blends and also clarification of the mechanism of phase separation.

The range of wavelength used in the light scattering experiments is limited so that we are not necessarily able to detect the dominant concentration fluctuation at the early stage SD. $\mathrm{X}$-Rays are sometimes used as a light source to observe segmental order fluctuation. Though small-angle X-ray scattering (SAXS) is favor- able from the point of detectable structural dimension, it generally requires long exposure time. So it is not suited for time-resolved measurements of structural change. As far as we know, only a few reports ${ }^{13-16}$ have been published on the application of SAXS for the dynamic study of phase separation of partially miscible blends. In these reports synchrotron radiation (SR) was used as the X-ray source because of its high flux, which is needed to perform time-resolved measurements. Phase separation phenomena were investigated for both upper critical solution temperature (UCST) type blends and lower critical solution temperature (LCST) type blends by time-resolved SR-SAXS: for the UCST type, low molecular weight polystyrene/polybutadiene blends, ${ }^{13}$ and for the LCST type, poly-

$\uparrow$ Present address: Sumitomo Naugatuck Co., Ltd., Tsukahara, Takatsuki, Osaka 561, Japan.

${ }_{\dagger \dagger}$ Present address: Sumitomo Chemical Co., Ltd., Ehime Research Laboratory, Niihama, Ehime 792, Japan. 
(methyl methacrylate) (PMMA)/ chlorinated polyethylene blends. ${ }^{15,16}$

This paper reports on the early stage of $\mathrm{SD}$ of PMMA/poly(styrene-co-acrylonitrile) (SAN) partially miscible blends which show LCST behavior by using time-resolved SRSAXS.

\section{EXPERIMENTAL}

The samples used here were commercially available PMMA $\left(M_{w}=91700, M_{w} / M_{n}=1.97\right)$ and SAN $\left(M_{w}=145200, M_{w} / M_{n}=2.07, \mathrm{AN}\right.$ content: $30.3 \mathrm{wt} \%)$. Molecular weight and chemical composition were determined by GPC and elemental analysis.

Blends were prepared by dissolving both PMMA and SAN in THF at a total concentration of $1 \mathrm{wt} \%$, and precipitating into excess $n$-heptane. Each sample specimen for SAXS measurement was made by hot-press molding into a sheet of $1 \mathrm{~mm}$ thickness at $135^{\circ} \mathrm{C}$ and annealed for 1 day at $135^{\circ} \mathrm{C}$.

Time-resolved SR-SAXS measurements were performed at the photon factory, National Laboratory for High Energy Physics using SAXS apparatus (BL-15A $\left.{ }^{17}\right)$. The wavelength of incident $\mathrm{X}$-rays was $0.1504 \mathrm{~nm}$, and the camera length was $2245 \mathrm{~mm}$. Scattering X-rays were detected by position sensitive proportional counter (PSPC) with efficient length of $170 \mathrm{~mm}$ and the covered wave number $(q)$ range was $0.06-1.5 \mathrm{~nm}^{-1}$. Time-resolved scattering intensities were collected by CAMAC histomemories controlled by PDP-11 computer.

A method of mixing heated and cooled nitrogen gases was adopted in order to achieve rapid temperature changes of specimens. Temperature in the sample stage was controlled by changing the mixing rate of heated and cooled nitrogen gases and heater temperature. Temperature in the sample stage could be raised rapidly at a rate of more than $250^{\circ} \mathrm{Cmin}^{-1}$.

Phase diagram of the blend system was obtained from cloud point measurement by extrapolation to zero temperature elevation after measurements with various temperature elevations $\left(1,2,4^{\circ} \mathrm{C} \mathrm{min}^{-1}\right)$.

\section{RESULTS AND DISCUSSION}

Figure 1 shows the LCST type phase diagram of this blend system. Temperature was elevated rapidly from the one-phase region ( $)$ into the two-phase region $(\boldsymbol{\Delta})$ as indicated by the arrows.

The typical time-course of temperature jump experiment is shown in Figure 2. Temperature was monitored on the spot very close to the specimens in the hot stage. The system reached the quench temperature in a short time, about 20 seconds in this case, which is rapid enough for detecting the slow phase separation of this blend system in our experimental temperature range. Total scattering intensity, $I_{\text {total }}$, increased rapidly after quenching, which means initiation of phase separation. Finally $I_{\text {total }}$ began to decrease owing to the shift of scattering peak into the direct beam stop. Here time was measured after the temperature became constant throughout the experiments.

Figure 3 depicts typical time-resolved SAXS

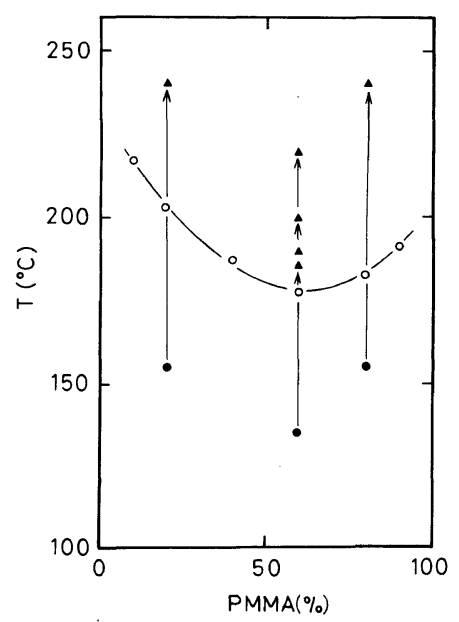

Figure 1. Phase diagram for PMMA/SAN blend obtained from cloud point measurements. The arrows indicate temperature jump from the one-phase region to the two-phase region. 


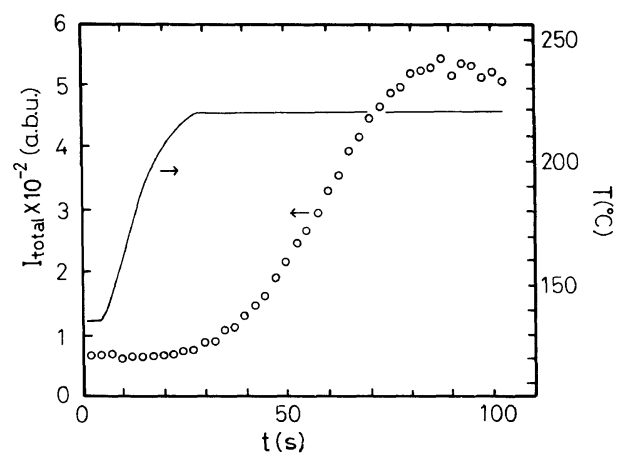

Figure 2. Typical time-course of temperature-jump experiment. Total scattering intensity, $I_{\text {total }}$, and temperature were plotted against time for PMMA/SAN (60/40) blend.

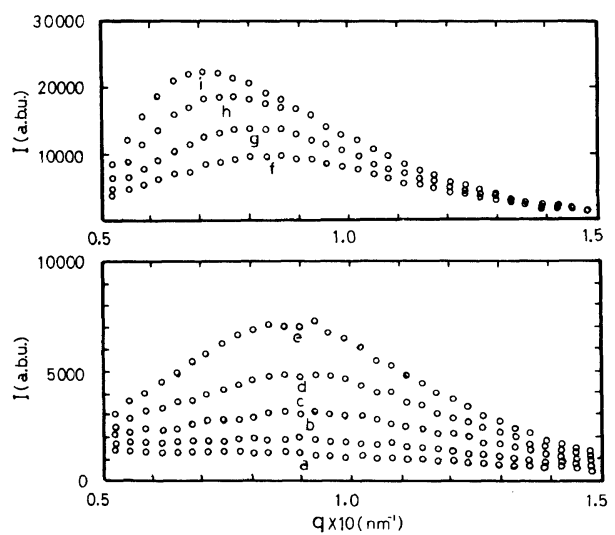

Figure 3. Time-resolved SR-SAXS profiles at $200^{\circ} \mathrm{C}$ for PMMA/SAN (60/40) blend at different times after temperature jump. a) $35 \mathrm{~s}$; b) $47 \mathrm{~s}$; c) $59 \mathrm{~s}$; d) $71 \mathrm{~s}$; e) $83 \mathrm{~s}$; f) $95 \mathrm{~s}$; g) $119 \mathrm{~s}$; h) $137 \mathrm{~s}$; i) $161 \mathrm{~s}$.

profiles of the $60 / 40$ blend, in which temperature jumped from $135^{\circ} \mathrm{C}$ to $200^{\circ} \mathrm{C}$. Typical behavior of SD reported for miscible blend systems were also observed in this blend system as follows;

(i) A scattering maximum appeared in a smaller $q$ range with the lapse of time,

(ii) subsequently the intensity of scattering maximum, $I_{\mathrm{m}}$, increased, but the wave number of scattering maximum, $Q_{\mathrm{m}}$, remained constant,

(iii) later, $Q_{\mathrm{m}}$ decreased and $I_{\mathrm{m}}$ increased at the same time.

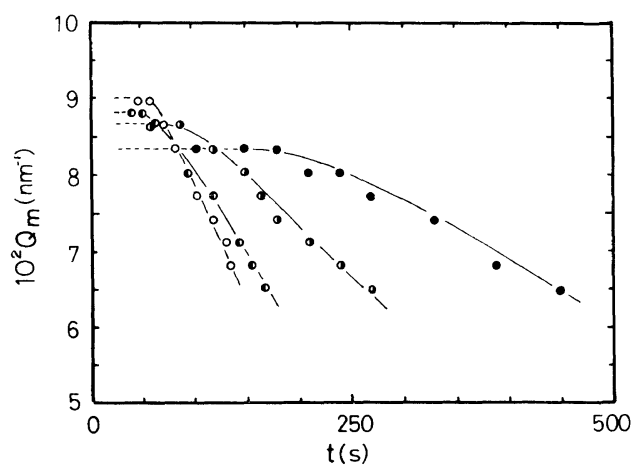

Figure 4. Variation of $Q_{\mathrm{m}}$ with time at different quench temperature for PMMA/SAN $(60 / 40)$ blend. Quench temperature: (O) $185^{\circ} \mathrm{C}$; (O) $190^{\circ} \mathrm{C}$; (O) $200^{\circ} \mathrm{C}$; (O) $220^{\circ} \mathrm{C}$.

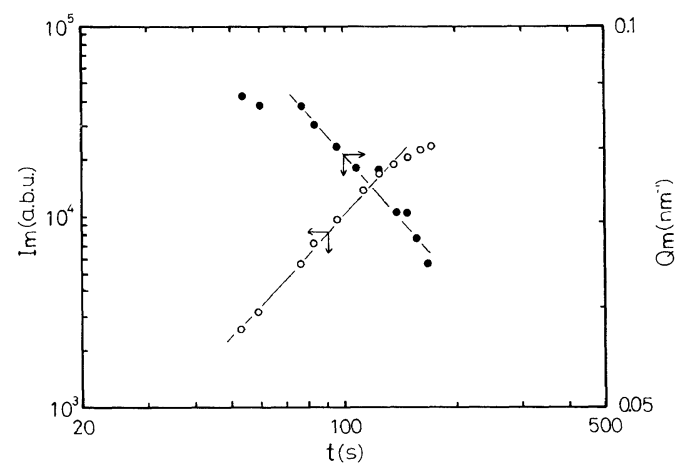

Figure 5. Typical $\log -\log$ plot of $I_{\mathrm{m}}$ and $Q_{\mathrm{m}}$ vs.t. Quench temperature is $200^{\circ} \mathrm{C}$.

These results mean that we could observe the early stage and transition from the early stage to the intermediate stage of phase separation.

Figure 4 illustrates $Q_{\mathrm{m}}$ variation with time at each quenching temperature. After the initial constant period, $Q_{\mathrm{m}}$ decreased with time corresponding to coarsening of structure. In Figure 4 , initial $Q_{\mathrm{m}}$ increased with the quenching temperature, but the variation was unexpectedly small compared to the other systems. $^{3,6}$

Figure 5 shows the typical log-log plot of $I_{\mathrm{m}}$ and $Q_{\mathrm{m}} v \mathrm{~s}$. time for the $60 / 40$ blend quenched at $200^{\circ} \mathrm{C}$ in the second stage following the first stage where $Q_{\mathrm{m}}$ is kept constant. In the scaling relations of $Q_{\mathrm{m}}$ and $I_{\mathrm{m}}\left(Q_{\mathrm{m}} \sim t^{-\alpha}, I_{\mathrm{m}} \sim t^{\beta}\right)$ it has 
Table I. $\alpha$ and $\beta$ values for PMMA/SAN $(60 / 40)$ blend quenched at various temperatures

\begin{tabular}{cccc}
\hline$T /{ }^{\circ} \mathrm{C}$ & $\alpha$ & $\beta$ & $\beta / \alpha$ \\
\hline 185 & 0.35 & 2.1 & 6.1 \\
190 & 0.36 & 2.0 & 5.5 \\
200 & 0.36 & 2.2 & 6.0 \\
220 & 0.38 & 2.8 & 7.5 \\
\hline
\end{tabular}

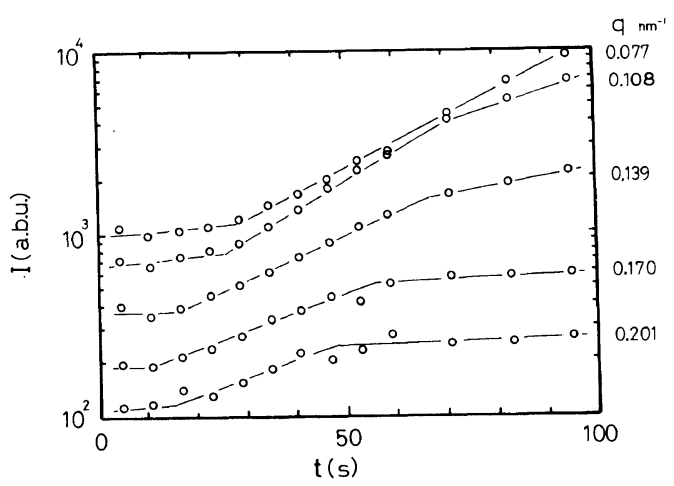

Figure 6. Variation of $I(q)$ with time at different wave vectors for PMMA/SAN (60/40) blend. Quench temperature is $200^{\circ} \mathrm{C}$.

been reported ${ }^{18}$ that $\beta>3 \alpha$ in the intermediate stage of spinodal decomposition and $\beta=3 \alpha$ in the late stage. As listed in Table I, $\beta>3 \alpha$ is fulfilled at each temperature, suggesting that the observed second stage is in the intermediate stage. The value of $\alpha$ was calculated from Figure 5 to be about $1 / 3$, which is larger than the value estimated from LBM theory. ${ }^{19}$

Figure 6 depicts scattering intensity $I(q)$ variation with time at each $q$, the magnitude of wave vector. $\log I(q)$ increased linearly with the time at first, which was followed by successive increase of slower rate during the period when $Q_{\mathrm{m}}$ changed with time (in Figure 4). Hence, the system probably has reached the intermediate stage here.

There existed an induction period only at small $q$, where $I(q)$ remained constant. Existence of the apparent induction time only at low $q$ may be attributed to the finite rate of temperature jump experiment.

Recently Okada et al. reported ${ }^{5}$ that the

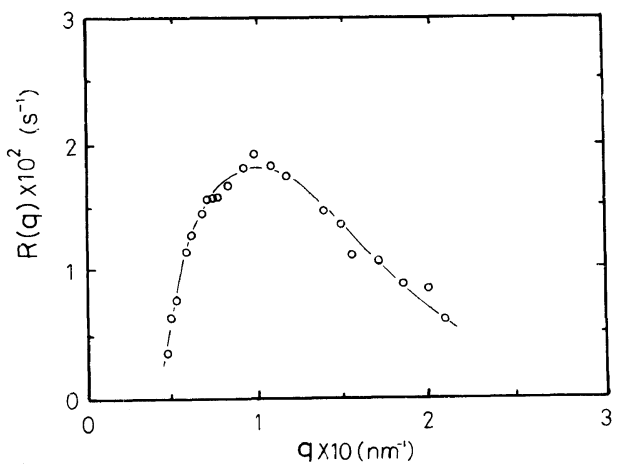

Figure 7. Dependence of the growth rate of concentration fluctuation, $R(q)$, on wave vector, $q$, for PMMA/SAN (60/40) blend. Quench temperature is $200^{\circ} \mathrm{C}$.

thermal noise term on scattering intensity is not negligible. According to their results, $I(q)$ is expressed in eq 1 , where $I_{\infty}$ is intensity corresponding to the virtual structure factor, $I_{0}$ is initial intensity and $R(q)$ is the growth rate of concentration fluctuation.

$$
I(q, t)=I_{\infty}+\left(I_{0}-I_{\infty}\right) \exp (2 R(q) t)
$$

Plot of $\log I(q, t)$ against $t$ is predicted not to be linear due to the thermal noise term, $I_{\infty}$, which is variable with quench temperature. The good linearity shown in Figure 6 for our system seems to be due to small $I_{\infty}$, which might be attributable to rather deep quenching (10 $40^{\circ} \mathrm{C}$ ). If $R(q)$ is large and $I_{\infty}$ is small, then eq 1 predicts exponential growth at sufficient large t. $R(q)$ was determined from eq 1 with substitution of $I_{\infty}$ to be zero.

Figure 7 depicts the dependence of $R(q)$ on $q$. $R(q)$ was maximum at the wave number of dominant concentration fluctuation, $Q_{\mathrm{m}}$, which is in the same order as that in Figure 3. Cahn's theory ${ }^{12}$ expressed as eq 2 is only applicable to $q$ range, $q \ll R_{\mathrm{g}}^{-1}$, where $R_{\mathrm{g}}$ denotes the radius of gyration of a polymer chain as pointed out by Binder. ${ }^{20}$

$$
R(q) / q^{2}=D_{\text {app }}\left(1-q^{2} / 2 Q_{\mathrm{m}}^{2}\right)
$$

where $D_{\text {app }}$ is the apparent diffusion constant. In the case of asymmetric polymer blend of polymer $\mathrm{A}$ and polymer $\mathrm{B}$, that is, $R_{\mathrm{g}, \mathrm{A}} \neq R_{\mathrm{g}, \mathrm{B}}$, 


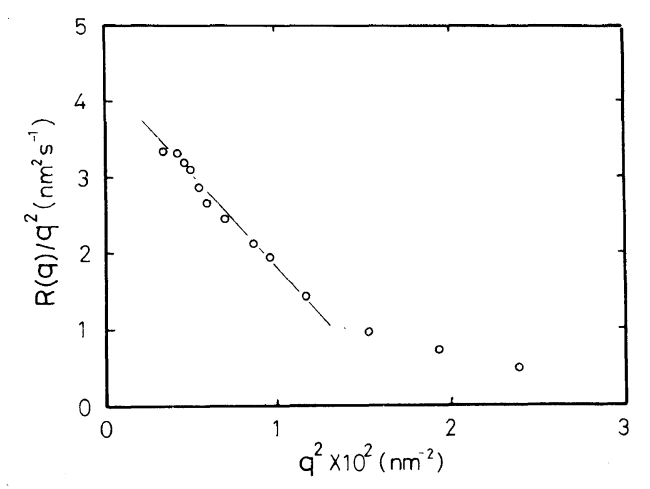

Figure 8. Plot of $R(q) / q^{2} v s . q^{2}$ for PMMA/SAN (60/40) blend at $200^{\circ} \mathrm{C}$.

Table II. $D_{\text {app }}$ and $Q_{\mathrm{m}}$ of PMMA/SAN belnd calculated from the Cahn-Hilliard theory at various conditions

\begin{tabular}{cccc}
\hline PMMA $/ \%$ & $T /{ }^{\circ} \mathrm{C}$ & $D_{\text {app }} / \mathrm{nm}^{2} \mathrm{~s}^{-1}$ & $10^{2} Q_{\mathrm{m}} / \mathrm{nm}^{-1}$ \\
\hline 80 & 240 & 19.7 & 6.48 \\
60 & 185 & 1.30 & 8.35 \\
60 & 190 & 3.06 & 8.82 \\
60 & 200 & 4.19 & 9.49 \\
60 & 220 & 4.30 & 9.50 \\
20 & 240 & 32.0 & 6.81 \\
\hline
\end{tabular}

average $R_{\mathrm{g}}$ is calculated by eq 3 .

$$
R_{\mathrm{g}}^{2}=\phi_{\mathrm{A}} R_{\mathrm{g}, \mathrm{A}}^{2}+\phi_{\mathrm{B}} R_{\mathrm{g}, \mathrm{B}}^{2}
$$

Here, $\phi_{\mathrm{i}}$ is the volume fraction and $R_{\mathrm{g}, \mathrm{i}}$ is the radius of gyration of component $i$. In this experiment, $R_{\mathrm{g}}$ was calculated to be 7 and $10 \mathrm{~nm}$ from $M_{w}$ and $M_{n}$ value, respectively, using unperturbed dimension in dilute solution data. $^{21}$

Only limited data covers the $q$ range of .$q \ll R_{\mathrm{g}}^{-1}$ in our experiment, but eq 2 was applied to this system as a first order approximation by using the data only in the range of $q<R_{\mathrm{g}}^{-1}$. From the intercept and slope of the plot of Figure $8, D_{\text {app }}$ and $Q_{\mathrm{m}}$ were determined to be $4.19 \mathrm{~nm}^{2} \mathrm{~s}^{-1}$ and $0.0949 \mathrm{~nm}^{-1}$, respectively.

The values of $Q_{\mathrm{m}}$ calculated from eq 2 were consistent with those of measured $Q_{\mathrm{m}}$ in Figure 3 , as listed in Table II together with the results of other blend ratios. $D_{\text {app }}$ were also calculated

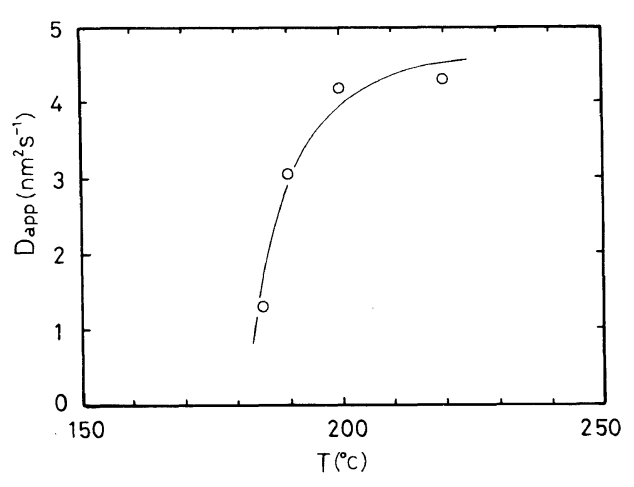

Figure 9. Dependence of $D_{\mathrm{app}}$ on quench temperature.

for the other quench temperature, and are shown in Figure 9. The values of $D_{\text {app }}$ decreased rapidly with decreasing temperatures, which suggests that the system approaches the phase boundary.

\section{CONCLUSION}

It was found that SR-SAXS technique enables us to detect the initial phase separation of a partially miscible blend on the order of tens of nanometers. The Cahn-Hilliard theory was successfully applied to the results obtained from SR-SAXS experiments.

We adopted approximations used in light scattering which provides information on a smaller $q$ range than SAXS to our system. In the larger $q$ range mainly observed here by SAXS, however, the conventional theory may not be necessarily adequate, which might reflect the curved plot in Figure 8. For example, the deviation from eq 2 at large $q$ is probably due to the error of estimating $R(q)$, that is, the substitution of $I_{\infty}$ as zero becomes invalid because $I_{\infty}$ is large when $q>Q_{\mathrm{c}}$, where $Q_{\mathrm{c}}$ is the $q$ at $R(q)=0$. Further, neglect of $q$ dependence of Onsager coefficient and the approximation of virtual structure factor by OrnsteinZernike form used in derivation of eq 2 might not be adequate in the larger $q$ range observed by SAXS as pointed out by Binder. ${ }^{20} \mathrm{We}$ should therefore analyze SAXS data by a 
theory valid in the large $q$ range in future. Small $Q_{\mathrm{m}}$ dependence on temperature near phase boundary as pointed out in other systems ${ }^{15,16}$ is not appropriate to Cahn's theory. Thus the theory seems to be inadequate in this aspect, but careful examination in temperature range much closer to the phase boundary must be done to get final conclusions.

Study on the molecular weight dependence of $D_{\mathrm{app}}$ is now in progress, which is interesting from the viewpoint of polymer chain dynamics.

\section{REFERENCES}

1. S. Nojima, Y. Ohyama, M. Yamaguchi, and T. Nose, Polym. J., 14, 907 (1982).

2. T. Hashimoto, J. Kumaki, and H. Kawai, Macromolecules, 16, 641 (1983).

3. H. L. Snyder and P. Meakin, J. Polym. Sci., Polym. Symp., 73, 217 (1985).

4. H. Yang, M. Shibayama, R. S. Stein, N. Shimidzu, and T. Hashimoto, Macromolecules, 19, 1667 (1986).

5. M. Okada and C. C. Han, J. Chem. Phys., 85, 5137 (1986).

6. J. G. Voight-Martin, K. H. Leister, R. Rosenau, and R. Konningsveld, J. Polym. Sci., B, 24, 723 (1986).
7. M. Takenaka, T. Izumitani, and T. Hashimoto, Macromolecules, 20, 2257 (1987).

8. T. Sato and C. C. Han, J. Chem. Phys., 88, 2057 (1988).

9. T. Kyu and J. M. Saldanha, Macromolecules, 21, 1021 (1988).

10. T. Inoue and T. Ougizawa, J. Macromol. Sci.-Chem., A26(1), 147 (1989).

11. H. Nakamura, J. Maruta, T. Ohnaga, and T. Inoue, Polymer, 31, 303 (1990).

12. J. W. Cahn, J. Chem. Phys., 42, 93 (1965).

13. T. P. Russell, G. Hadziioannou, and W. K. Warburton, Macromolecules, 18, 78 (1985).

14. G. R. Strobl, Macromolecules, 18, 558 (1985).

15. J. S. Higgins, H. Fruitwala, and P. E. Tomlins, $B r$. Polym. J., 21, 247 (1989).

16. J. S. Higgins, H. Fruitwala, and P. E. Tomlins, Macromolecules, 22, 3674 (1989).

17. Y. Amemiya, K. Wakabayashi, T. Hamanaka, T. Wakabayashi, T. Matsushita, and H. Hashizume, Nucl. Instrum. Methods, 208, 471 (1983).

18. T. Hashimoto, M. Itakura, and N. Shimidzu, $J$. Chem. Phys., 85, 6773 (1986).

19. J. S. Langer, M. Bar-on, and H. D. Miller, Phys. Rev., A11, 1417 (1975).

20. K. Binder, J. Chem. Phys., 79, 6387 (1983).

21. J. Brundrup and E. H. Immergut, "Polymer Handbook," Interscience, New York, N.Y., 1965. 